\title{
Theory of time-resolved inelastic x-ray diffraction
}

\author{
Ulf Lorenz, $^{*}$ Klaus B. Møller, ${ }^{\dagger}$ and Niels E. Henriksen ${ }^{\ddagger}$ \\ CMM, Department of Chemistry, Building 207, Technical University of Denmark, DK-2800 Kgs. Lyngby, Denmark
}

(Received 23 October 2009; published 24 February 2010)

\begin{abstract}
Starting from a general theory of time-resolved x-ray scattering, we derive a convenient expression for the diffraction signal based on a careful analysis of the relevant inelastic scattering processes. We demonstrate that the resulting inelastic limit applies to a wider variety of experimental conditions than similar, previously derived formulas, and it directly allows the application of selection rules when interpreting diffraction signals. Furthermore, we present a simple extension to systems simultaneously illuminated by $\mathrm{x}$ rays and a laser beam.

DOI: 10.1103/PhysRevA.81.023422

PACS number(s): $34.80 . \mathrm{Qb}, 32.80 . \mathrm{Wr}, 33.80 .-\mathrm{b}, 42.50 . \mathrm{Ct}$
\end{abstract}

\section{INTRODUCTION}

Since its discovery, x-ray diffraction has become a major tool for studying the structure of matter. Its key virtue is that, in a time-independent framework [1], the differential cross section for elastic scattering is just the Fourier transform of the electronic density $\varrho_{e}$,

$$
\frac{d \sigma}{d \Omega}=\left|\int d^{3} r \varrho_{e}(\mathbf{r}) e^{i \mathbf{q r}}\right|^{2},
$$

where we neglected the weak scattering from the nuclei and expressed the cross section in units of the scattering of a free electron. This gives almost direct access to real-space information of the electronic structure. In contrast to optical photons, $x$ rays interact with all electrons, which makes the cross section insensitive to minor variations of valence orbitals. One can therefore often go further and use the independent atom model (IAM), in which one models $\varrho_{e}$ by the density of the isolated atoms. Within this approximation, the diffraction pattern becomes a function of the atomic positions in the probed system.

It is therefore not surprising that currently, much work is spent on the development of intense, pulsed, femtosecond $\mathrm{x}$-ray sources, namely, free electron lasers (FELs). After exciting a system with an optical pump pulse, the subsequent dynamics can be followed in time by a femtosecond x-ray pulse with variable time delay. If the time-resolved scattering can be described by a formula similar to Eq. (1), this allows us to directly follow atomic rearrangements without the need for complex electronic structure calculations that link the experimental signal to the molecular dynamics. However, similar to modern ultrafast optical pump-probe experiments, we require an extension of the conventional static theory.

The previous work on the theoretical foundations of timeresolved $\mathrm{x}$-ray diffraction (TRXD) can be roughly divided into two categories. In one approach, the conventional expression (1) is used as the starting point [2-8]. Essentially, the electronic density gets an additional time variable, and the result is convoluted with the time-dependent intensity of the x-ray beam to obtain the signal as a function of the delay time. However, Eq. (1) is typically derived from Fermi's Golden

\footnotetext{
*ulf.lorenz@kemi.dtu.dk

${ }^{\dagger}$ klaus.moller@kemi.dtu.dk

†neh@kemi.dtu.dk
}

Rule $[1,6]$. In the derivation of the Golden Rule, the initial state is assumed to be an eigenstate of the unperturbed Hamiltonian, that is, to have no intrinsic time dependence. An alternative derivation of (1) uses classical electromagnetic theory for the treatment of the x-ray field [4]. Here, it is assumed that the electric current in the target system is exclusively induced by the incoming $\mathrm{x}$-ray field, which again requires a negligible time evolution. Furthermore, the (classical) x-ray field and the (quantum) target system are coupled via the dielectric constant. This quantity, however, is obtained from time-independent perturbation theory and can therefore be assigned only to time-independent states, not to wave packets. Strictly speaking, formulas obtained by these derivations are thus not valid for describing ultrafast, coherent wave-packet motion. However, they have been applied successfully to subpicosecond TRXD experiments [8], which suggests that, though the derivation is different, the formulas for TRXD should have a form similar to that of the time-independent theory.

In another approach, one evaluates the interaction between the studied nonstationary quantum system and the $\mathrm{x}$-ray photon field, usually within first-order perturbation theory. This has been done with a wave-packet $[9,10]$ or density-operator formalism [11-13].

In general, the interaction between the $\mathrm{x}$ rays and the target system can be either elastic, leaving the state of the target systems unchanged, or inelastic, thereby changing the population of the individual energy eigenstates. With the exception of $[12,13]$, previous work focused on a hybrid "electronically elastic" scattering. It was assumed that the scattering process is inelastic with respect to nuclear modes but elastic with respect to electronic states, or that contributions to electronically inelastic diffraction can be removed by special experimental setups. It is interesting to note that the same approximation of electronically elastic scattering has also been used in the closely related field of time-resolved electron diffraction (e.g., [14]). However, this approach is different from the earliest treatments on X-ray diffraction [15], which explicitly considered electronically inelastic components. In this paper, we want to review the theory for TRXD without such a hybrid scheme. We obtain a less restrictive, more general formulation, which also permits the use of symmetry arguments in the evaluation of the diffraction signal.

In Sec. II, we outline and discuss the general systemindependent formulation. We then focus on molecular systems in Sec. III, and connect our results to those derived previously. 
Section IV summarizes our findings and provides an outlook on further research. In the appendices, we discuss an extension of the theory to systems with time-dependent Hamiltonians, and discuss in more detail some of the approximations used in the derivations.

\section{GENERAL THEORY}

The key quantity of interest in x-ray diffraction is the differential signal strength $d S / d \Omega$, which we define as the number of scattered photons arriving in a certain solid angle. If we allow these photons to have frequencies $\omega_{s}$ that are different from the central frequency $\omega_{0}$ of the incoming beam, we can write

$$
\frac{d S}{d \Omega}=\int_{\omega_{1}}^{\omega_{2}} d \omega_{s} \frac{d^{2} S}{d \Omega d \omega_{s}}
$$

to express it in terms of the double-differential signal strength. This quantity is then defined as

$$
\frac{d^{2} S}{d \Omega d \omega_{s}}=\rho\left(\omega_{s}\right) \lim _{t \rightarrow \infty}\left\langle\Xi_{0}\left|\hat{U}^{\dagger}\left(t, t_{0}\right) \hat{n}_{\mathbf{k}_{s}, \epsilon_{s}} \hat{U}\left(t, t_{0}\right)\right| \Xi_{0}\right\rangle .
$$

The initial state $\Xi_{0}$ is a direct product consisting of a target in a prepared nonstationary state $\Psi$ and a photon state $\psi_{u k_{0}}$ that describes the x-ray pulse on its way to the target. They interact, and after essentially infinite time, the scattered photons hit a detector that is modeled by the photon number operator $\hat{n}_{\mathbf{k}_{s}, \epsilon_{s}}$, and counts the number of photons with wave vector $\mathbf{k}_{s}$ and optionally polarization $\epsilon_{s}$. Multiplication by the density of photon states $\rho$ yields the number of scattered photons per solid angle. If the propagation is carried out with first-order perturbation theory for the interaction between the target and the $\mathrm{x}$ rays, we obtain (see [10] for the details)

$$
\frac{d^{2} S}{d \Omega d \omega_{s}}=\left(\frac{d \sigma}{d \Omega}\right)_{\mathrm{Th}} \frac{\omega_{s}}{\omega_{0}} s\left(\mathbf{q}, \omega_{0}-\omega_{s}\right),
$$

where

$$
\begin{aligned}
& s(\mathbf{q}, \omega)=\frac{1}{2 \pi} \int d t I(t) \int d \delta C(\delta) e^{i \omega \delta} \\
& \quad \times\left\langle\Psi\left(t+\frac{\delta}{2}\right)\left|\hat{L}^{\dagger} \hat{U}\left(t+\frac{\delta}{2}, t-\frac{\delta}{2}\right) \hat{L}\right| \Psi\left(t-\frac{\delta}{2}\right)\right\rangle .
\end{aligned}
$$

Here, $(d \sigma / d \Omega)_{\mathrm{Th}}$ is the classical Thomson cross section of a free electron,

$$
I(t)=\frac{2 \epsilon_{0} c}{\hbar \omega_{0}}\left\langle\psi_{u k_{0}}\left|\hat{E}^{(-)} \hat{E}^{(+)}\right| \psi_{u k_{0}}\right\rangle
$$

is the photon number intensity of the incoming beam,

$$
\hat{L}=\sum_{i=1}^{\text {\#electrons }} e^{i \mathbf{q} \hat{\mathbf{r}}_{i}}
$$

is the scattering operator with $\mathbf{q}=\mathbf{k}_{\mathbf{0}}-\mathbf{k}_{\mathbf{s}}$ being the scattering vector, and

$$
C(\delta)=\int d \omega^{\prime} F\left(\omega_{0}+\omega^{\prime}\right) e^{i \omega^{\prime} \delta}
$$

defines the coherence function of the x-ray beam in terms of the normalized power spectrum $F$, which is centered around $\omega_{0}$.
The typical decay time $T_{c}$ of the coherence function is inversely proportional to the width $\Delta \omega_{0}$ of $F$. Note that the convention for some of the symbols differs from [10] for convenience, and to be more in line with the classic literature.

As discussed in [10], the result is also valid for the important practical case where the X-ray beam is not a coherent photon state but described by incoherent ensembles. This result was derived using two important assumptions. We assumed that the beam is fully characterized by its intensity and power spectrum. This only holds for special cases, such as FELs in the linear regime [16] or ensembles of identical Gaussian pulses. Furthermore, we have neglected wave-vector dispersion of the x-ray beam. In the classical limit, this translates to the substitution of the electric field

$$
\mathbf{E}(\mathbf{r}, t)=\mathbf{E}_{0} h(\mathbf{r}, t) e^{i\left(\mathbf{k}_{0} \mathbf{r}-\omega_{0} t\right)} \approx \mathbf{E}_{0} h(\mathbf{R}, t) e^{i\left(\mathbf{k}_{0} \mathbf{r}-\omega_{0} t\right)},
$$

that is, the envelope function $h$ is only evaluated at the position $\mathbf{R}$ of the target system. The incoming beam is thus assumed to have a fixed wave vector $\mathbf{k}_{\mathbf{0}}$, but varying frequencies. The idea is that the wave vector of the incoming photon only enters through the scattering vector $\mathbf{q}$, whose variations can be neglected. We discuss this approximation in the next subsection when we also vary the length of the wave vector of the scattered photon, $\mathbf{k}_{s}$.

In the following, we will discuss the limit of inelastic diffraction in more detail, using an analysis similar to the work of Cao and Wilson [9]. For more convenient manipulation, we start by transforming (5) to an energy eigenstate representation. If the Hamiltonian $\hat{H}$ of the probed system is time independent (for time-dependent systems, see Appendix A), the wave function can be expanded as

$$
\Psi(t)=\sum_{i} c_{i} \varphi_{i} e^{-i E_{i} t / \hbar}, \quad \hat{H} \varphi_{i}=E_{i} \varphi_{i} .
$$

Inserting this and performing standard manipulations yields

$$
\begin{aligned}
s\left(\mathbf{q}, \omega_{0}-\omega_{s}\right)= & \int d t I(t) \sum_{i j k} c_{i}^{*} c_{j} e^{-i\left(E_{j}-E_{i}\right) t / \hbar} L_{k i}^{*} L_{k j} \\
& \times F\left(\omega_{s}+\frac{1}{\hbar}\left(E_{k}-\frac{E_{i}+E_{j}}{2}\right)\right),
\end{aligned}
$$

with $L_{i k}=\left\langle\varphi_{i}|\hat{L}| \varphi_{k}\right\rangle$.

\section{A. Inelastic limit}

Let us first consider the limit of elastic diffraction $L_{i j}=$ $\delta_{i j} L_{i i}$. Equation (11) simplifies to

$$
s\left(\mathbf{q}, \omega_{0}-\omega_{s}\right)=\sum_{i}\left|c_{i}\right|^{2}\left|L_{i i}\right|^{2} F\left(\omega_{s}\right) \int d t I(t),
$$

and the diffraction image becomes independent of time. The reason is that the interference terms $i \neq j$ in (11) hold the information about the time evolution of the quantum system, but they do no contribute here. We conclude thus that we are not interested in purely elastic scattering, but we have to include inelastic terms. 
To do so, we insert (11) and (4) into (2) and change the order of integration and summation, which yields

$$
\begin{aligned}
\frac{d S}{d \Omega}= & \left(\frac{d \sigma}{d \Omega}\right)_{\mathrm{Th}} \int d t I(t) \sum_{i j k} c_{i}^{*} c_{j} e^{-i\left(E_{j}-E_{i}\right) t / \hbar} \\
& \times \int_{\omega_{1}}^{\omega_{2}} d \omega_{s} L_{k i}^{*} L_{k j} \frac{\omega_{s}}{\omega_{0}} F\left(\omega_{s}+\frac{1}{\hbar}\left(E_{k}-\frac{E_{i}+E_{j}}{2}\right)\right) .
\end{aligned}
$$

This expression is difficult to simplify because the summation over $i, j, k$, and the integration over $\omega_{s}$ are entangled. This happens through the argument of $F$, but also through the scattering operator $\hat{L}$, since $\mathbf{k}_{s}$, and thus $\mathbf{q}$, is a function of the frequency $\omega_{s}$. However, this problem is not unique to time-resolved diffraction. For systems in their stationary ground state, $c_{i}=c_{j}=\delta_{i 0}$, Eq. (13) corresponds to timeindependent diffraction, and the entanglement of the remaining $k$ summation and the $\omega_{s}$ integration is still in there. In this context, a solution for this problem is well known under the name "static approximation" $[15,17]$. To get an insight into the approximations and limitations when applying this to TRXD, we shall go through the derivation in some detail.

The probed target system is in a nonstationary state whose energy spread is at most a few eV, that is, $\left|E_{i}-E_{j}\right| \ll \hbar \omega_{0}$. Furthermore, the basis of the static approximation is the assumption that the matrix elements $L_{i k}$ can be neglected unless $\left|E_{k}-E_{i}\right| \ll \hbar \omega_{0}$, which we discuss in detail in Appendix B. Thus, although we formally retain the unrestricted sums over $i, j, k$, only those summands with $|\Delta E|=$ $\left|E_{k}-\left(E_{i}+E_{j}\right) / 2\right| \ll \hbar \omega_{0}$ contribute to the signal.

To exploit this relation, we require the interval $\left[\omega_{1}, \omega_{2}\right]$ to include $\omega_{0}$. We also assume that the power spectrum $F$ has a not too large width $\Delta \omega_{0} \ll \omega_{0}$. The integrand is then dominated by contributions with $\left|\omega_{s}-\omega_{0}\right| \lesssim|\Delta E| / \hbar+$ $\Delta \omega_{0} \ll \omega_{0}$, otherwise, either $F$ or the matrix elements $L_{k i}$, $L_{k j}$ vanish.

As a result of this, we find that the scattering vector remains almost constant when integrating over $\omega_{s}$. For example, we find from geometric relations that

$$
\begin{aligned}
& \left|\frac{q^{2}}{q_{0}^{2}}-1\right| \leqslant\left|\frac{\Delta E}{\hbar \omega_{0}}\right|+\left|\frac{\Delta \omega_{0}}{\omega_{0}}\right|^{2}+\left|\frac{\Delta \omega_{0} \Delta E}{\hbar \omega_{0}^{2}}\right| \\
& +\left(\frac{|\Delta E|+2 \hbar \Delta \omega_{0}}{\hbar c q_{0}}\right)^{2},
\end{aligned}
$$

where $q_{0}$ is the magnitude of the scattering vector for elastic scattering $k_{s}=k_{0}$, and $c$ is the speed of light. For typical FEL parameters $\hbar \omega_{0} \approx 10 \mathrm{keV}, \Delta \omega_{0} / \omega_{0}=10^{-3}$ [18], and assuming $|\Delta E| \leqslant 100 \mathrm{eV}$ (see Appendix B), and $q_{0}=$ $0.5-8 \AA^{-1}$, the relative variation $\left|q / q_{0}\right|$ is less than $3 \%$.

Since the length of the scattering vector stays almost constant, and diffraction patterns are usually not overly sensitive to the value of $\mathbf{q}$ (see, e.g., examples in [1] and discussion in [10]), we now replace the scattering operator $\hat{L}$ by the elastic version $\hat{L}_{0}=\hat{L}\left(\mathbf{q} \mid k_{s}=k_{0}\right)$, and move its matrix elements outside of the integral. Using all the approximations so far, and setting $\omega_{s} / \omega_{0} \approx 1$, the remaining integral

$$
\int_{\omega_{1}}^{\omega_{2}} d \omega_{s} F\left(\omega_{s}+\frac{1}{\hbar}\left(E_{k}-\frac{E_{i}+E_{j}}{2}\right)\right)
$$

gives a constant of unity for all $i, j, k$, if $\omega_{1} \rightarrow 0$ and $\omega_{2} \rightarrow \infty$, i.e., if the detector collects all photons. We can then rewrite the differential signal strength as

$$
\frac{d S}{d \Omega}=\left(\frac{d \sigma}{d \Omega}\right)_{\mathrm{Th}} \int d t I(t)\left\langle\Psi(t)\left|\hat{L}_{0}^{\dagger} \hat{L}_{0}\right| \Psi(t)\right\rangle .
$$

This is the inelastic limit of x-ray diffraction. A similar expression has been derived in the context of TRXD before $[9,10]$. However, the previous works do not stress its fundamental importance for time-resolved diffraction. As our more detailed derivation shows, (16) is the relevant limit if the static approximation is valid, and the following experimental conditions hold:

1. The system evolves freely during the scattering process.

2. Only states with low energies are excited, that is, $\mid E_{i}-$ $E_{j} \mid \ll \hbar \omega_{0}$, which should be fulfilled for all but a few exotic experiments.

3. The incoming $x$-ray beam has a sufficiently narrow bandwidth $\Delta \omega_{0} \ll \omega_{0}$.

4. We collect all scattered photons, $\omega_{1} \rightarrow 0, \omega_{2} \rightarrow \infty$.

Since these resemble typical experimental conditions, we conclude that the inelastic limit should be generally chosen as the starting point for further theory. This limit also has properties that are of special importance in the context of time-resolved diffraction.

First, it is not restricted to a special representation of the wave function. This allows us to use the same basic formula (16) for different tasks such as mapping atomic orbitals [2] or measuring atomic rearrangements within a molecule. We can also trivially recast the result using a density operator $\hat{\varrho}$. If we neglect decoherence (i.e., statistical mixing with a thermal bath) during the interaction with the $\mathrm{x}$-ray pulse, we can write directly

$$
\frac{d S}{d \Omega}=\left(\frac{d \sigma}{d \Omega}\right)_{\mathrm{Th}} \int d t I(t) \operatorname{Tr}\left[\hat{\varrho}(t) \hat{L}_{0}^{\dagger} \hat{L}_{0}\right] .
$$

For completeness, we want to point out that a more detailed derivation would show that this density-operator formulation holds as long as we can neglect decoherence during the coherence time of the X-ray pulse, which is at most about a femtosecond [19]. For this, we first rewrite (5) with a density operator [see also Eq. (42) in [12]]. If decoherence can be neglected during the coherence time, an expansion equivalent to (10) can be performed. The rest of the discussion about the static approximation only involved matrix elements of $\hat{L}$, and it is equally valid for all representations of the quantum state.

The only restriction we had to put on the target system is a time-independent Hamiltonian. By examining carefully why and how we did this, we can find a simple extension of the theory to systems irradiated by lasers if the laser parameters change sufficiently slowly. This is detailed in Appendix A.

Finally, the operator $\hat{L}_{0}^{\dagger} \hat{L}_{0}$ is symmetric under space inversion, which allows the use of symmetry arguments in the evaluation of expectation values. This is in contrast to the operator $\hat{L}$ that has no trivial symmetry properties. 


\section{B. Symmetry considerations}

In many experiments, we study systems whose total Hamiltonian is symmetric under space inversion, for example, free molecules in the gas phase. The energy eigenstates, which are typically the initial states for the pump-probe experiments, also obey this symmetry. If we assume that spin is a good quantum number, they are either even or odd functions with respect to the inversion of all (nuclear and electronic) space coordinates.

The same argument holds for systems in contact with a thermal bath, such as small sections in a liquid sample. In thermal equilibrium, the density operator of the system commutes with the Hamiltonian $[\hat{H}, \hat{\rho}]=0$. In the absence of degeneracies, and for Hamiltonians symmetric under inversion, each eigenstate of this density operator (or, equivalently, each member of the thermal ensemble) is then an even or odd function of the coordinates.

The excitation of the system with a linearly polarized pump laser involves the dipole operator $\hat{\mu}$, which is odd under space inversion. It is known from elementary group theory that each application then changes the symmetry of the state. If we excite the system by absorption of an odd number of photons, we thus create a coherent superposition of a ground state $\Psi_{g}$ and an excited state wave packet $\Psi_{e}$

$$
|\Psi(t)\rangle=\left|\Psi_{g}(t)\right\rangle+\left|\Psi_{e}(t)\right\rangle,
$$

with $\Psi_{g}, \Psi_{e}$ transforming differently under space inversion. When calculating (16), contributions of the form

$$
\left\langle\Psi_{g}(t)\left|\hat{L}_{0}^{\dagger} \hat{L}_{0}\right| \Psi_{e}(t)\right\rangle=\left\langle\Psi_{g}(t)\left|\sum_{i \neq j}^{N_{e}} e^{i \mathbf{q}\left(\hat{\mathbf{r}}_{i}-\hat{\mathbf{r}}_{j}\right)}\right| \Psi_{e}(t)\right\rangle
$$

are zero, because the operator $\hat{L}_{0}^{\dagger} \hat{L}_{0}$ is invariant under space inversion. The diffraction image is thus an incoherent sum of the images of the ground and excited state wave function.

However, this argument does not hold under certain circumstances:

1. If the Hamiltonian is not symmetric under inversion, the eigenstates have no symmetry properties, and the previous argument is void. The typical example here are molecules at interfaces.

2. For systems that are oriented prior to the arrival of the pump pulse, the symmetry of the initial state is destroyed by the orientation.

3. If the excitation includes an even number of photons, $\Psi_{g}, \Psi_{e}$ have the same symmetry properties.

In these cases, the interference terms (19) also show up in the diffraction pattern, which is now the coherent sum of the two states. We thus have the possibility to include or exclude specific contributions to the signal.

\section{APPLICATION TO MOLECULES}

We use the well-known Born-Huang representation [20] to apply the theory to molecules. For this, we decompose the system into nuclei and electrons and denote their collective coordinates as $\mathbf{R}$ and $\mathbf{r}$. The total wave function is factorized into "nuclear" and "electronic" wave functions

$$
\Psi(\mathbf{R}, \mathbf{r}, t)=\sum_{m} \Lambda_{m}(\mathbf{R}, t) \lambda_{m}(\mathbf{r} ; \mathbf{R}) .
$$

For each $\mathbf{R}$, the electronic states $\lambda_{m}$ are chosen to form an orthonormal basis set in the electronic subsystem

$$
\left\langle\lambda_{m}(\mathbf{R}) \mid \lambda_{n}(\mathbf{R})\right\rangle_{r}=\delta_{m n} .
$$

Here and in the following, we use the subscript $r$ to denote an integration over only the electronic coordinates. Furthermore, we will drop the explicit $\mathbf{R}$ dependence of $\lambda_{m}$. For most applications, only a few electronic states need to be considered, which greatly reduces the dimensionality of the quantum system.

We can directly insert the Born-Huang representation (20) into (16) and obtain the diffraction signal of a molecule. Writing out the integration over the nuclear coordinates explicitly, we obtain

$$
\begin{aligned}
\frac{d S}{d \Omega}= & \left(\frac{d \sigma}{d \Omega}\right)_{\mathrm{Th}} \int d t I(t) \sum_{n, m} \\
& \times \int d \mathbf{R} \Lambda_{m}^{*}(\mathbf{R}, t) \Lambda_{n}(\mathbf{R}, t) s_{m n}(\mathbf{R}), \\
& s_{m n}(\mathbf{R})=\left\langle\lambda_{m}\left|\hat{L}_{0}^{\dagger} \hat{L}_{0}\right| \lambda_{n}\right\rangle_{r} .
\end{aligned}
$$

For density operators, the Born-Huang representation corresponds to the expansion

$$
\hat{\varrho}(t)=\sum_{m n} \hat{\varrho}_{m n}(t)\left|\lambda_{n}\right\rangle\left\langle\lambda_{m}\right|
$$

where the operators $\hat{\varrho}_{m n}$ act on the nuclear degrees of freedom only. We write out the trace over the electronic degrees of freedom explicitly and evaluate the trace over the nuclear coordinates in a local basis. Equation (17) becomes then

$$
\begin{gathered}
\frac{d S}{d \Omega}=\left(\frac{d \sigma}{d \Omega}\right)_{\mathrm{Th}} \int d t I(t) \sum_{m, n} \int d \mathbf{R} \varrho_{m n}(\mathbf{R}, t) s_{m n}(\mathbf{R}) \\
\varrho_{m n}(\mathbf{R}, t)=\left\langle\mathbf{R}\left|\hat{\varrho}_{m n}(t)\right| \mathbf{R}\right\rangle .
\end{gathered}
$$

The terms $\varrho_{m m}(\mathbf{R})$ are the nuclear densities and define the probability density of finding a nuclear configuration $\mathbf{R}$ with a certain electronic state $\left|\lambda_{m}\right\rangle$. The nondiagonal terms $\varrho_{m n}(\mathbf{R})$ are the nuclear coherences; the corresponding terms describe interferences between the different electronic states in the diffraction pattern.

For practical use, and to gain more insight, we shall now consider additional approximations to connect the general result (25) to those derived previously [9-11].

\section{A. Connection to other derivations}

While the results so far are applicable to an arbitrary electronic basis $\lambda_{m}$, we now restrict our choice to the adiabatic basis. If we write the Hamiltonian as a sum

$$
\hat{H}=\hat{T}_{R}+\hat{h}
$$

of the kinetic energy $\hat{T}_{R}$ of the nuclei and an "electronic Hamiltonian" $\hat{h}$, the adiabatic basis are those electronic states 
that diagonalize $\hat{h}$. This is a common choice and has been selected by other authors we wish to compare our results to.

We start by having a closer look at the coherences $\varrho_{m n}(\mathbf{R})$. For slow nuclear motion, they oscillate with a frequency of roughly the inverse electronic energy spacing. If the length of the X-ray pulse and the timing jitter between the pump and probe pulses are small enough to resolve these oscillations, the coherences show up as "beating" patterns in the diffraction image.

However, these terms can often be neglected. For wellseparated electronic states, the time resolution needs to be on the order of single femtoseconds to measure them. If one of the states $m, n$ is populated through excitation from the other state by absorbing an odd number of photons, the coherence terms between these two states will not contribute due to the symmetry arguments outlined earlier. Most importantly, we argue in Appendix B that for many systems, the matrix elements $s_{m n}$ are significantly smaller than the diagonal terms $s_{m m}$, thus reducing the contribution from the coherences.

If we drop the contributions of the nuclear coherences, only a single sum in (25) is left. To simplify the evaluation of the remaining matrix elements $s_{m m}(\mathbf{R})$, we use a resolution of identity $\mathbb{1}_{r}=\sum_{n}\left|\lambda_{n}\right\rangle\left\langle\lambda_{n}\right|$ to formally separate them into an elastic and a purely inelastic contribution

$$
s_{m m}=\left|\left\langle\lambda_{m}\left|\hat{L}_{0}\right| \lambda_{m}\right\rangle_{r}\right|^{2}+\sum_{n \neq m}\left|\left\langle\lambda_{n}\left|\hat{L}_{0}\right| \lambda_{m}\right\rangle_{r}\right|^{2} .
$$

Inserting this into (25) yields, after neglecting the nuclear coherence terms,

$$
\begin{aligned}
\frac{d S}{d \Omega}= & \left(\frac{d \sigma}{d \Omega}\right)_{\mathrm{Th}} \int d t I(t) \sum_{m} \\
& \times \int d \mathbf{R} \varrho_{m m}(\mathbf{R}, t)\left|\left\langle\lambda_{m}\left|\hat{L}_{0}\right| \lambda_{m}\right\rangle_{r}\right|^{2}+S_{\text {inel }} .
\end{aligned}
$$

The first term gives the "electronically elastic" scattering and has a simple interpretation. The matrix element $\left\langle\lambda_{m}\left|\hat{L}_{0}\right| \lambda_{m}\right\rangle$ is the Fourier transform of the electronic density of state $m$. Thus, the first term is similar to the standard result of $\mathrm{X}$-ray diffraction [see Eq. (1)] weighted by a time-averaged distribution of nuclear geometries. $S_{\text {inel }}$ denotes the purely inelastic contribution to the signal. Its interpretation and evaluation is much more cumbersome, so we wish to remove it in one way or the other.

Two solutions have been proposed in the literature. In one approach [5], it is assumed that the inelastic contribution can be neglected because the nondiagonal elements $\left\langle\lambda_{n}\left|\hat{L}_{0}\right| \lambda_{m}\right\rangle_{r}$ are small compared to the diagonal terms. This general result stems from the fact that only a single orbital contributes to the matrix element (see Appendix B), while all electrons contribute to the elastic scattering. However, this argument is problematic because, even though the single matrix elements are small (see Appendix B), the sum in (28), and thus $S_{\text {inel }}$ includes many terms. For atoms, this inelastic scattering contribution has been tabulated (e.g., [21]). Even for heavy species, such as iodine, the elastic and inelastic contributions are similar in magnitude for $q \gtrsim 6 \AA^{-1}$, so this seems too crude an approximation.

Other references suggested an energy-scale separation (vaguely described in [9], implicitly used in [10], and explicitly pointed out in [11]). For this, we note that (11) only gives a significant contribution if the argument of $F$ is approximately $\omega_{0}$. The intuitive picture that emerges is that each excitation of the system leads to a corresponding energy loss of the outgoing photon. For a nearly monochromatic source, $F(\omega) \approx \delta\left(\omega-\omega_{0}\right)$, the energy of the outgoing photons exhibits a peak for each resonance. We can now use the Born-Oppenheimer approximation, and we assume that energy levels corresponding to an excitation of nuclear modes (which we wish to include) have much smaller energy spacing than the electronic states (which we want to remove). By filtering out the latter contributions (i.e., photons deviating from $\omega_{0}$ by more than, say, $1 \mathrm{eV}$ ) before they reach the detector, we can remove all electronically inelastic components from the diffraction pattern, so that $S_{\text {inel }}=0$.

However, this argument has several problems as well. The energy-scale separation becomes meaningless for close-lying electronic states. Thus, this line of thought excludes nonadiabatic transitions, which are very common in photochemistry. Furthermore, the required excessive filtering of the incoming and outgoing photons might reduce the signal by orders of magnitude, which makes this setup somewhat unattractive.

However, we note that practical experiments often use difference diffraction techniques for TRXD (e.g., [4,22]). That is, two diffraction images are taken, one with the pump laser turned on and off, respectively, and the two signals are subtracted to yield a difference signal. If we are interested in the difference signal, we do not have to require that the inelastic contribution vanishes. If it is independent of the atomic arrangement, and the electronic state, the corresponding terms cancel already due to norm conservation. Furthermore, in many experiments, the scattering signal is evaluated using the independent atom model (IAM) (e.g., $[1,9,10])$ for the description of the electronic densities. The IAM approximates the electronic structure of a molecule by that of its independent constituent atoms. It does therefore not describe the reorganization of electronic densities due to chemical binding. As detailed in Appendix B, we can show that $S_{\text {inel }}$ is roughly independent of the molecular structure within the limit of the IAM, and it can thus be ignored in difference diffraction images. A similar argument has been used previously for the case of gas-phase electron diffraction by Liu and Lin [23]. This solution allows us to approximate the scattering with molecular targets as being electronically elastic, solves all of the problems mentioned earlier, but still allows us to employ, for example, symmetry rules derived in the inelastic limit.

We finally assume that the scattering from all electronic states is similar, as only a few valence orbitals are rearranged, and replace the matrix elements by the IAM form factor (see, e.g., $[9,10]$, and Appendix B3)

$$
\left\langle\lambda_{m}\left|\hat{L}_{0}\right| \lambda_{m}\right\rangle_{r} \approx f_{\mathrm{IAM}}(\mathbf{q} ; \mathbf{R})=\sum_{\alpha}^{\text {\#atoms }} f_{\alpha}(\mathbf{q}) e^{i \mathbf{q} \mathbf{R}_{\alpha}},
$$

with the atomic form factors

$$
f_{\alpha}(\mathbf{q})=\int d^{3} r \varrho_{\alpha}(\mathbf{r}) e^{i \mathbf{q r}},
$$

and $\varrho_{\alpha}$ and $\mathbf{R}_{\alpha}$ the ground-state electronic density and position of atom $\alpha$. Since we deal with difference patterns, we 
further denote by $\Delta \varrho_{m m}$ the difference densities between our diffraction pattern and a reference pattern (e.g., obtained by turning the excitation laser off). Equation (29) gives

$$
\begin{aligned}
\frac{d \Delta S}{d \Omega}= & \left(\frac{d \sigma}{d \Omega}\right)_{\mathrm{Th}} \int d t I(t) \\
& \times \int d \mathbf{R}\left[\sum_{m} \Delta \varrho_{m m}(\mathbf{R}, t)\right]\left|f_{\mathrm{IAM}}(\mathbf{q} ; \mathbf{R})\right|^{2}
\end{aligned}
$$

The quantity in brackets is the difference in the probability density of finding a certain nuclear geometry $\mathbf{R}$ at time $t$. Within the limit of the IAM and neglect of coherence terms, the diffraction signal is therefore just the product of the "time-averaged difference distribution of geometries," $\int I(t) \sum_{m} \Delta \varrho_{m m}(\mathbf{R}, t) d t$, and the IAM form factor, integrated over all geometric configurations $\mathbf{R}$. This final result closely resembles the time-independent theory but with a distribution function that depends on the pump-probe delay time.

\section{CONCLUSIONS AND OUTLOOK}

In this paper, we reviewed the general result of our previous work on time-resolved $\mathrm{x}$-ray diffraction for nonstationary systems [10] and reformulated it in a form that is more in line with the standard results for time-independent systems.

We argued that in the context of scattering on nonstationary systems, it is essential to allow for inelastic scattering processes in the target system but that the scattering operator only connects states whose energy difference is small compared to the energy of the incoming photons (the "static approximation"). Furthermore, under typical experimental conditions, we can replace the scattering operator by its elastic version. This leads to the central result for the differential signal strength given in Eq. (16).

We applied this formalism to molecules and obtained expressions that contain nuclear coherences between different electronic states. This implies that the diffraction patterns are not equal to the classical incoherent sum of signals from each of the involved electronic states, but involve fast-oscillating "beating" contributions [9]. We argued, however, that in many situations, these coherences can be neglected, even for closely spaced electronic states. Employing further results from the widely used independent atom model, the calculation of difference diffraction patterns can be condensed into a simple and appealing form, Eq. (32).

Having established the formalism allows future investigation of new questions. It is, for example, well known that wave packets created by ultrashort laser pulses can show strong dispersion. In contrast to vibrational ground states typically encountered in stationary systems, these wave packets can easily span distances of several Ångströms. The impact of this on the obtainable information from diffraction patterns is basically unknown. Furthermore, we argued that the nuclear coherence terms can be usually neglected. For systems composed of light atoms, however, they might show up in diffraction patterns. Identifying promising systems and describing the resulting diffraction patterns requires a more detailed quantitative analysis.

\section{ACKNOWLEDGMENTS}

This work was supported by the Danish National Research Foundation's Center for Molecular Movies (CMM). We thank Stephan P. A. Sauer for useful discussions.

\section{APPENDIX A: SYSTEMS WITH TIME-DEPENDENT HAMILTONIAN}

In the course of our formal derivations, we required that the system evolves under a time-independent Hamiltonian, Eq. (10). In this appendix, we review this restriction. As it turns out, this restriction can be relaxed to encompass systems driven by a laser with slowly varying parameters. We first employ Floquet theory to formally remove the periodic oscillation of the electric field, then we use the adiabatic theorem to treat nonperiodic (pulsed) laser fields.

The limit of an overlapping laser and x-ray pulse is important for two reasons. First, by illuminating molecules with a laser, we can create nonequilibrium structures that can then be probed by the x-ray beam. For example, it has been proposed to align gas-phase molecules with a laser while scattering off them [11]. Since the distribution of molecular orientations is then no longer isotropic, this would yield additional information about the structure. Second, we find that the final result has the same basic form as in the case of time-independent Hamiltonians, so if the x-ray pulse and the excitation pulse happen to overlap, the corresponding diffraction images do not need to be discarded or treated in any special way.

\section{Floquet theory}

Within semiclassical theory and electric-dipole approximation, the Hamiltonian of a system under the influence of a continuous-wave laser with amplitude $\varepsilon_{0}$ is

$$
\hat{H}(t ; \theta)=\hat{H}_{0}+\hat{\mu} \varepsilon_{0} \cos (\omega t+\theta),
$$

where $\hat{H}_{0}$ is the time-independent Hamiltonian of the unperturbed system, and $\hat{\mu}$ the dipole operator. Our goal is to transform this time-dependent problem into a time-independent description by using Floquet theory [24-27]. As the basic idea, the Schrödinger equation with Hamiltonian (A1) is solved for all initial phases $\theta$ simultaneously. For this, one first defines an enlarged Floquet space [26]

$$
\mathcal{K}=\mathcal{H} \otimes L_{2}\left(\mathbb{S}^{1} ; d \theta / 2 \pi\right)
$$

as the direct product of the original Hilbert space $\mathcal{H}$ and the space of square-integrable, $2 \pi$-periodic functions of $\theta$. This space is equipped with a natural scalar product

$$
\langle\langle\xi \mid \eta\rangle\rangle=\frac{1}{2 \pi} \int_{0}^{2 \pi} d \theta\langle\xi(\theta) \mid \eta(\theta)\rangle .
$$

The single brackets $\langle\mid\rangle$ denote the scalar product in $\mathcal{H}$. We now lift the initial state that we wish to propagate, $\varphi_{0}(x)=\varphi\left(x, t_{0}\right)$, to $\mathcal{K}$ by

$$
\xi_{0}(\theta)=\varphi_{0} \otimes \mathbb{1}_{\theta}
$$

and propagate $\xi_{0}$ such that

$$
\varphi\left(x, t ; \theta_{0}\right) \equiv \xi\left(x, \theta_{0}+\omega\left(t-t_{0}\right), t\right)
$$


is the solution when propagating $\varphi_{0}$ with the Hamiltonian $\hat{H}\left(t ; \theta_{0}\right)$ from (A1). It can be shown [26] that the propagator $\hat{U}_{K}$ of $\xi$ is generated by a time-independent Floquet Hamiltonian

$$
\hat{K}(\theta)=\hat{H}(t=0 ; \theta)-i \hbar \omega \partial_{\theta} .
$$

The time dependence of the original Hamiltonian is hidden in the mapping $\mathcal{K} \rightarrow \mathcal{H}$ for fixed initial phase. To formally remove this time dependence, we can evaluate expectation values directly in $\mathcal{K}$. Any operator $\hat{A}$ that is independent of the phase $\theta$ can be trivially lifted to the Floquet space. Calculating the expectation value then gives

$$
\langle\langle\xi(t)|\hat{A}| \xi(t)\rangle\rangle=\frac{1}{2 \pi} \int_{0}^{2 \pi} d \theta\langle\varphi(t ; \theta)|\hat{A}| \varphi(t ; \theta)\rangle .
$$

Thus, by using Floquet theory, we can formally remove any periodic time dependence in the Hamiltonian at the cost of an integration over all initial phases. The scattering of $x$-ray photons is described by the scattering operator $\hat{L}$, which is not correlated with the excitation laser, so Eq. (5) can be retained with all scalar products evaluated in $\mathcal{K}$, and propagators replaced by the Floquet propagators $\hat{U}_{K}$.

This leaves open the problem of evaluating the phase average (A7). Qualitatively, the averaging removes laserinduced oscillations $\sim \cos n \omega t(n \in \mathbb{Z})$, and is therefore often referred to as averaging over a period of the laser (e.g., [28], where the derived Hamiltonian can also be obtained from Floquet perturbation theory [25]).

\section{Adiabatic Hamiltonians}

Floquet theory itself removes only the periodic time dependence. In practice, however, we encounter lasers with a time-dependent amplitude and possibly a chirp, which are not strictly periodic. In this case, we can separate out the central frequency as periodicity and write the Hamiltonian in (A1) with a time-dependent field strength $\varepsilon_{0}=\varepsilon_{0}(t)$. While we can still evaluate Eq. (5) in the Floquet space, and thereby remove oscillations $\sim \cos n \omega t$, the Floquet Hamiltonian is now time-dependent $\hat{K}(\theta, t)$. This leads to a problem, because the discussion leading to the inelastic result (16) revolved around an expansion of the wave function in the basis of energy eigenstates.

To solve this problem, we make use of the adiabatic theorem [29]. For this, we first expand the wave function in the basis of instantaneous eigenstates of $\hat{K}$ in analogy to (10)

$$
\left.|\Psi(t)\rangle\rangle=\sum_{i} c_{i}(t)\left|\varphi_{i}(t)\right\rangle\right\rangle
$$

with

$$
\left.\left.\hat{K}(t)\left|\varphi_{i}(t)\right\rangle\right\rangle=E_{i}(t)\left|\varphi_{i}(t)\right\rangle\right\rangle .
$$

The transformation to an eigenstate basis was done by inserting various resolutions of identity into (5). The instantaneous eigenstates also form a complete set of eigenstates, so we can do the same here to obtain

$$
\begin{aligned}
& \sum_{i j k l} c_{i}^{*}(t) c_{j}(t)\left\langle\left\langle\varphi_{i}(t)\left|\hat{U}_{K}\left(t, t+\frac{\delta}{2}\right) \hat{L}^{\dagger}\right| \varphi_{k}\left(t+\frac{\delta}{2}\right)\right\rangle\right\rangle \\
& \quad \times\left\langle\left\langle\varphi_{k}\left(t+\frac{\delta}{2}\right)\left|\hat{U}_{K}\left(t+\frac{\delta}{2}, t-\frac{\delta}{2}\right)\right| \varphi_{l}\left(t-\frac{\delta}{2}\right)\right\rangle\right\rangle \\
& \quad \times\left\langle\left\langle\varphi_{l}\left(t-\frac{\delta}{2}\right)\left|\hat{L} \hat{U}_{K}\left(t-\frac{\delta}{2}, t\right)\right| \varphi_{j}(t)\right\rangle\right\rangle .
\end{aligned}
$$

If the laser parameters, and thus the Floquet Hamiltonian $\hat{K}(t)$ change slowly in time with respect to the coherence time of the beam (i.e., the value of $\delta$ ), we can use the adiabatic approximation [29]

$$
\begin{aligned}
\left.\hat{U}_{K}(t \pm \delta, t)\left|\varphi_{i}(t)\right\rangle\right\rangle & \left.\approx\left|\varphi_{i}(t \pm \delta)\right\rangle\right\rangle e^{\mp i E_{i}(t) \delta / \hbar} \\
& \left.\approx\left|\varphi_{i}(t)\right\rangle\right\rangle e^{\mp i E_{i}(t) \delta / \hbar}
\end{aligned}
$$

Inserting (A10) and (A11) into (5) yields

$$
\begin{aligned}
s\left(\mathbf{q}, \omega_{0}-\omega_{s}\right)= & \int d t I(t) \sum_{i j k} c_{i}^{*}(t) c_{j}(t) \\
& \times\left\langle\left\langle\varphi_{i}(t)\left|\hat{L}^{\dagger}\right| \varphi_{k}(t)\right\rangle\right\rangle\left\langle\left\langle\varphi_{k}(t)|\hat{L}| \varphi_{j}(t)\right\rangle\right\rangle \\
& \times F\left(\omega_{s}+\frac{1}{\hbar}\left[E_{k}(t)-\frac{E_{i}(t)+E_{j}(t)}{2}\right]\right),
\end{aligned}
$$

which is just Eq. (11) lifted to the Floquet space, and with time-dependent energies and states. If the intensity of the laser is not too strong, and the photon energy not too high, we can expect the instantaneous Floquet states to be "wellbehaved" and fulfill all important restrictions (e.g., the static approximation). All derivations can be performed as in the time-independent case with all expectation values or traces evaluated in $\mathcal{K}$. Especially, Eqs. (17) and (32) maintain their form and interpretation.

Note, however, that formally all eigenstates in (A12) have to change adiabatically, those that are occupied as well as those that can be occupied due to inelastic scattering events. Because of the energy scales involved in these scattering events, the adiabatic approximation (A11) cannot be rigorously validated in practice but has to be assumed to hold. Also, we want to point out that although (A12) uses a formally inconvenient time-dependent expansion of the wave function, the final representation-independent result (16) still holds.

$\mathrm{X}$-ray scattering from laser-aligned molecules has been described previously by Ho and Santra [11], who implicitly used adiabatic Floquet theory by employing the alignment Hamiltonian of Friedrich and Herschbach [28]. As our formal approach demonstrates, for the special cases of periodic and adiabatic perturbations, the scattering theory is not different from that for time-independent Hamiltonians. Furthermore, we want to point out that because of our more general formulation of the diffraction process, we do not need any further assumptions about the Hamiltonian as in [11]; specifically, the formalism is equally valid for resonant and nonresonant laser parameters. 


\section{APPENDIX B: QUALITATIVE EVALUATION OF MATRIX ELEMENTS}

In this appendix, we review the static approximation, consider the electronic cross terms of Eq. (23), and discuss how the basic ideas of the IAM can simplify the evaluation of the inelastic matrix elements in Eq. (28). We use the formalism of second quantization [30] and drop nuclear and spin coordinates for easier notation. Given a single-electron basis $\phi_{p}$, denoting the usual creation and annihilation operators in this basis as $\hat{a}_{p}^{\dagger}$ and $\hat{a}_{p}$, and the vacuum state as $|0\rangle$, the set of Slater determinants

$$
\left|\Phi_{\mathbf{I}}\right\rangle=\hat{a}_{I_{1}}^{\dagger} \cdots \hat{a}_{I_{N_{e}}}^{\dagger}|0\rangle
$$

forms an orthonormal basis in the space of $N_{e}$ electrons. We can expand electronic states in this basis

$$
\left|\lambda_{m}\right\rangle=\sum_{\mathbf{I}} c_{\mathbf{I}}^{(m)}\left|\Phi_{\mathbf{I}}\right\rangle .
$$

The scattering operators can be written as [30]

$$
\begin{gathered}
\hat{L}_{0}=\sum_{p q} L_{p q} \hat{a}_{p}^{\dagger} \hat{a}_{q}, \\
\hat{L}_{0}^{\dagger} \hat{L}_{0}=N_{e}+\sum_{p q r s} L_{p q r s} \hat{a}_{p}^{\dagger} \hat{a}_{r}^{\dagger} \hat{a}_{s} \hat{a}_{q},
\end{gathered}
$$

with

$$
\begin{gathered}
L_{p q}=\int d^{3} x \phi_{p}^{*}(\mathbf{x}) e^{i \mathbf{q} \mathbf{x}} \phi_{q}(\mathbf{x}), \\
L_{p q r s}=L_{p q} L_{s r}^{*} .
\end{gathered}
$$

We suppress the summand $N_{e}$ in (B4) in the following because it only gives rise to a homogeneous background signal. We also suppress the subindex 0 from the scattering operators unless explicitly referring to the elastic scattering operator.

\section{Static approximation}

In the course of the derivation of the inelastic limit, we have assumed that matrix elements $\left\langle\lambda_{k}|\hat{L}| \lambda_{i}\right\rangle$ are zero unless the energy difference $\left|E_{k}-E_{i}\right|$ is sufficiently small. This static approximation can be deduced from qualitative considerations and inferred from somewhat limited experimental and theoretical data (e.g., [31-35]).

For the qualitative discussion, we assume that each energy eigenstate is made up of a single Slater determinant. Using the second quantization prescriptions (B1) and (B3), we obtain for two different eigenstates or Slater determinants

$$
\left\langle\Phi_{\mathbf{K}}|\hat{L}| \Phi_{\mathbf{I}}\right\rangle= \begin{cases}L_{k i}, & \text { if }\left|\Phi_{\mathbf{K}}\right\rangle=\hat{a}_{k}^{\dagger} \hat{a}_{i}\left|\Phi_{\mathbf{I}}\right\rangle, \\ 0, & \text { otherwise, }\end{cases}
$$

since the single-particle operator $\hat{L}$ can only change one electronic orbital at a time. We can thus restrict the discussion to properties of the single-electron basis. As a side note, we want to point out that each of these inelastic matrix elements is typically much smaller than the corresponding elastic term $\left\langle\Phi_{\mathbf{K}}|\hat{L}| \Phi_{\mathbf{K}}\right\rangle$. That is because Eq. (B7) only contains contributions from a single electron, while the elastic matrix element is the sum of the scattering from all occupied orbitals.

If we choose $E_{\mathbf{I}}<E_{\mathbf{K}}$, the orbital $\phi_{i}$ typically represents a bound state, while $\phi_{k}$ is some excited orbital. If $E_{\mathbf{K}}-E_{\mathbf{I}}$ is much larger than the binding energy $E_{i}$ of the bound (core or valence) orbital $\phi_{i}$, then $\phi_{k}$ is essentially a plane wave with kinetic energy $p^{2} / 2 m_{e} \sim E_{\mathbf{K}}-E_{\mathbf{I}}-E_{\text {ion }}$. The matrix element

$$
L_{k i}=\int d^{3} r e^{i(\mathbf{p}+\mathbf{q}) \mathbf{r}} \phi_{i}(\mathbf{r})
$$

is the high-momentum component of the bound orbital $\phi_{i}$, which decays exponentially on a typical scale $\propto \sqrt{m_{e} E_{\mathrm{ion}}}$. As the density of states only grows with the energy according to a power law ( $\propto E^{1 / 2}$ for plane waves), the contribution of states $\phi_{k}$ to the summation in (13) decreases exponentially in the limit of high energies.

In the extreme case, the vectors $\mathbf{q}$ and $\mathbf{p}$ can point in opposite directions, from which we obtain a qualitative estimate for the cutoff

$$
\left|E_{\mathbf{K}}-E_{\mathbf{I}}\right|_{\max }=\frac{q^{2}}{2 m_{e}}+2 E_{i}
$$

For typical values of $q$, the right-hand side of Eq. (B9) varies from tens to a few hundreds of eV. However, we can obtain more accurate and reliable values from experimental data or more elaborate calculations. As these are usually done for the ground state, we have to assume tacitly that the results will not change drastically for optically excited states.

The matrix elements $\left|\left\langle\lambda_{k}|\hat{L}| \lambda_{0}\right\rangle\right|^{2}$ occur in the context of generalized oscillator strengths (e.g., [36] and references therein) and are observables for inelastic scattering methods, such as electron energy loss spectroscopy [31]. However, the focus is often not on convergence properties, and the experimental data are somewhat scattered. Nevertheless, the available literature (e.g., [31-35]) also suggests a cutoff of at most a few $100 \mathrm{eV}$, which decreases for smaller values of $q$.

\section{Nondiagonal matrix elements of $\hat{L}_{0}^{\dagger} \hat{L}_{0}$}

After we introduced molecular systems, we rapidly dropped the coherence terms that connect different electronic states. Here, we want to discuss the specific argument that nondiagonal terms $s_{m n}$ defined in (23) are much smaller than the diagonal terms $s_{m m}$.

We first calculate the matrix elements of $\hat{L}_{0}^{\dagger} \hat{L}_{0}$ for single Slater determinants. The result is

$$
\left\langle\Phi_{\mathbf{K}}\left|\hat{L}_{0}^{\dagger} \hat{L}_{0}\right| \Phi_{\mathbf{I}}\right\rangle= \begin{cases}\sum_{q, r, q \neq r} L_{q q r r}-L_{q r r q}, & \left|\Phi_{\mathbf{K}}\right\rangle=\left|\Phi_{\mathbf{I}}\right\rangle, \\ \sum_{q \neq i} L_{k i q q}+L_{q q k i}-L_{k q q i}-L_{q i k q}, & \left|\Phi_{\mathbf{K}}\right\rangle=\hat{a}_{k}^{\dagger} \hat{a}_{i}\left|\Phi_{\mathbf{I}}\right\rangle, \quad k \neq i, \\ L_{k_{1} i_{1} k_{2} i_{2}}+L_{k_{2} i_{2} k_{1} i_{1}}-L_{k_{1} i_{2} k_{2} i_{1}}-L_{k_{2} i_{1} k_{1} i_{2},}, & \left|\Phi_{\mathbf{K}}\right\rangle=\hat{a}_{k_{1}}^{\dagger} \hat{a}_{k_{2}}^{\dagger} \hat{a}_{i_{2}} \hat{a}_{i_{1}}\left|\Phi_{\mathbf{I}}\right\rangle, \quad k_{1 / 2} \neq i_{1 / 2}, \\ 0, & \text { otherwise, }\end{cases}
$$


where the sums run over all occupied orbitals of determinant $\Phi_{\mathbf{I}}$. From this equation, we can deduce that the sum for the case $\mathbf{K}=\mathbf{I}$ contains $N_{e}^{2}$ terms, while for different determinants, we only sum at most over $N_{e}$ terms. This can be understood intuitively because at least one pair of creation and annihilation operators has to lift an electron from an orbital occupied in $\Phi_{\mathbf{I}}$ to another one solely occupied in $\Phi_{\mathbf{K}}$.

We can then calculate the nondiagonal matrix elements for the electronic states, which might consist of multiple determinants (B2). We also introduce two further approximations based on (B10):

1. If we assume that the matrix $L_{p q r s}$ is well balanced, matrix elements $\left\langle\Phi_{\mathbf{K}}\left|\hat{L}_{0}^{\dagger} \hat{L}_{0}\right| \Phi_{\mathbf{I}}\right\rangle$ with $\mathbf{I}=\mathbf{K}$ will be significantly larger than for different determinants. Under this assumption, we can choose to retain only matrix elements that involve the same determinants $\mathbf{I}, \mathbf{K}$. The error that we introduce by this approximation should then compare to the signal as $1 / N_{e}$.

2. We assume that all Slater determinants give the same contribution $\left\langle\Phi_{\mathbf{I}}\left|\hat{L}_{0}^{\dagger} \hat{L}_{0}\right| \Phi_{\mathbf{I}}\right\rangle=F$. The underlying idea is that all important Slater determinants differ only by a rearrangement of a few valence electrons, which is insignificant compared to the contribution of the core electrons. Correspondingly, we would expect the error to be of the order of the ratio between the number of valence electrons and the total number of electrons.

With these approximations, we obtain

$$
\begin{aligned}
s_{m n} & =\left\langle\lambda_{m}\left|\hat{L}_{0}^{\dagger} \hat{L}_{0}\right| \lambda_{n}\right\rangle=\sum_{\mathbf{I}, \mathbf{K}} c_{\mathbf{K}}^{(m) *} c_{\mathbf{I}}^{(n)}\left\langle\Phi_{\mathbf{K}}\left|\hat{L}_{0}^{\dagger} \hat{L}_{0}\right| \Phi_{\mathbf{I}}\right\rangle \\
& =F \sum_{\mathbf{I}} c_{\mathbf{I}}^{(m) *} c_{\mathbf{I}}^{(n)}=0,
\end{aligned}
$$

since the latter expression is just the scalar product between the two orthogonal electronic states.

We can therefore expect the nondiagonal elements to be small compared to the diagonal contributions $s_{m m}$ unless the approximations made earlier fail. The approximations might fail especially for molecules built of light atoms, which have a large fraction of valence electrons.

\section{Independent atom model}

The IAM is usually used in the context of elastic scattering. To apply it to the inelastic contributions, we first point out the underlying approximations in more detail. For simplicity, we ignore rotational averaging of the single atomic shells. To simplify notation, we also drop the index of the electronic state.

Obviously, as a first step, we have to assign the electrons to specific atoms. For this, we assume that the single-electron Hilbert space can be split up into subspaces $\mathcal{H}_{i}$ that are large enough to hold the wave function of the isolated atom $i$. We also assume that all these subspaces can be chosen to be orthogonal.

It should be pointed out that these two requirements are mutually exclusive. For practical molecular geometries, orbitals of different atoms have nonzero overlap. The corresponding subspaces can then not be chosen to be orthogonal and contain the wave function of the isolated atoms. However, assuming nonorthogonal subspaces significantly complicates the following algebra, and as we discuss at the end of this section, this overlap must be negligible for most orbitals anyway if the IAM is valid, so we will ignore this detail for simplicity.

We now introduce an orthonormal basis $\left\{\phi_{p}\right\}_{p \in \mathbb{N}}$, where the orbital $\phi_{p}$ shall belong to the subspace or atom $\mathcal{H}_{i}$ if $p$ is the element of some set $S_{i} \subseteq \mathbb{N}$. The orbitals are understood to have a fixed form and to move around with their respective atoms. That is,

$$
\phi_{p}(\mathbf{r})=g_{p}\left(\mathbf{r}-\mathbf{R}_{i}\right), \quad p \in S_{i},
$$

where $\mathbf{R}_{i}$ is the position of the $i$ th atom, and $g_{p}$ is the fixed functional form of the atomic orbital.

Next, we define states of the independent atoms as

$$
\left|\xi_{i}\right\rangle=\hat{C}_{i}^{\dagger}|0\rangle
$$

where $\hat{C}_{i}^{\dagger}$ is a (sum of) strings of creation operators $\hat{a}_{p}^{\dagger}\left(p \in S_{i}\right)$. The electronic structure of the independent atom $i$ shall be independent of the position of the atom, which requires $\hat{C}_{i}^{\dagger}$ to be independent of $\mathbf{R}_{i}$ as well.

With the notation fixed, the IAM can now be formulated as

$$
|\lambda\rangle \approx\left|\lambda^{\prime}\right\rangle=\left(\prod_{i=1}^{N_{a}} \hat{C}_{i}^{\dagger}\right)|0\rangle .
$$

Here, $N_{a}$ is the number of atoms in the molecule. Writing out the scattering amplitude and using the orthonormality of the atomic subspaces, we can bring this approximation into a more instructive form

$$
\left\langle\lambda\left|\hat{L}_{0}\right| \lambda\right\rangle \approx \sum_{i=1}^{N_{a}}\left\langle\xi_{i}\left|\hat{L}_{0}\right| \xi_{i}\right\rangle,
$$

that is, the molecular form factor [left-hand side of (B15)] is the sum of the atomic form factors, where we have not factored out the atomic coordinates explicitly. Within the IAM, we thus neglect electronic correlations between different atoms, which would lead to atomic cross terms.

Some identities that may help with the preceding and further manipulations are

$$
\begin{aligned}
L_{p q}\left(\mathbf{R}_{i}\right) & =L_{p q}\left(\mathbf{R}_{i}=0\right) e^{i \mathbf{q} \mathbf{R}_{i}}, & & p, q \in S_{i}, \\
{\left[\hat{C}_{i}^{\dagger}, \hat{a}_{p}\right]_{+} } & =\hat{C}_{i}^{\dagger} \hat{a}_{p}+\hat{a}_{p} \hat{C}_{i}^{\dagger}=0, & & p \notin S_{i}, \\
{\left[\hat{C}_{i}^{\dagger}, \hat{C}_{j}\right]_{+} } & =0, & & i \neq j .
\end{aligned}
$$

The first identity uses (B12) to factor out the geometrydependent complex phase from matrix elements; the second and third relations follow from the orthogonality of the atomic subspaces.

We now employ (B4), (B5), and (B14) to evaluate directly the matrix elements $s_{m m}$ in Eq. (28):

$$
\begin{aligned}
\left\langle\lambda\left|\hat{L}_{0}^{\dagger} \hat{L}_{0}\right| \lambda\right\rangle \approx & \left\langle\lambda^{\prime}\left|\hat{L}_{0}^{\dagger} \hat{L}_{0}\right| \lambda^{\prime}\right\rangle \\
= & \left(\sum_{i} \sum_{p, q, r, s \in S_{i}}+\sum_{i \neq j} \sum_{\substack{p, q \in S_{i} \\
r, s \in S_{j}}}+\sum_{i \neq j} \sum_{\substack{p, s \in S_{i} \\
r, q \in S_{j}}}\right) \\
& \times L_{p q} L_{s r}^{*}\left\langle\lambda^{\prime}\left|\hat{a}_{p}^{\dagger} \hat{a}_{r}^{\dagger} \hat{a}_{s} \hat{a}_{q}\right| \lambda^{\prime}\right\rangle,
\end{aligned}
$$


where we used the orthogonality relation (B17). It ensures that only those matrix elements contribute, where the creation operators act on the same subspaces as the annihilation operators.

The first summand in (B19) only involves orbitals around a single atom. The geometry-dependent phases of $L_{p q}$ and $L_{s r}^{*}$ then cancel, so that this contribution is independent of the nuclear geometry.

The second summand can be shown to be identical to the elastic term $\left|\left\langle\lambda^{\prime}\left|\hat{L}_{0}\right| \lambda^{\prime}\right\rangle\right|^{2}$ [i.e., the first term in (28)] apart from geometry-independent contributions. To demonstrate this, use (B15) together with (B17), and compare this to the second summand in (B19) after insertion of (B14) and extensive use of the commutation relations (B17) and (B18).

The last summand in (B19) contains matrix elements of orbitals around different atoms. It is therefore sensitive to the nuclear geometry but cannot be evaluated without detailed knowledge of the orbital shapes and the electronic structure of the single atoms. However, if it would contribute appreciably to the diffraction pattern, then matrix elements of the form (B6) and thus products $\phi_{p}^{*}(\mathbf{x}) \phi_{q}(\mathbf{x})$ with $p \in S_{i}, q \in S_{j}$ have to be significant for many occupied orbitals. In this case, mutual Coulomb repulsion between electrons of different atoms is also significant, leading to strong correlations between many electrons of different atoms and hence to a breakdown of the IAM. If the IAM level of theory is sufficient to fit the elastic part of Eq. (29), these correlations can be neglected, usually because the scattering is dominated by tightly bound, uncorrelated core electrons. As a consequence, the third summand in (B19) can then be neglected for the purpose of $\mathrm{X}$-ray diffraction. The inelastic part of the diffraction pattern $S_{\text {inel }}$ in Eq. (29) is thus roughly independent of the nuclear geometry.
[1] J. Als-Nielsen and D. McMorrow, Elements of Modern X-Ray Physics (Wiley, New York, 2001).

[2] M. Ben-Nun, T. J. Martínez, P. M. Weber, and K. R. Wilson, Chem. Phys. Lett. 262, 405 (1996).

[3] M. Ben-Nun, J. Cao, and K. R. Wilson, J. Phys. Chem. A 101, 8743 (1997).

[4] S. Bratos, F. Mirloup, R. Vuilleumier, and M. Wulff, J. Chem. Phys. 116, 10615 (2002).

[5] T. Rozgonyi, R. Sauerbrey, and T. Feurer, J. Appl. Phys. 97, 013537 (2005).

[6] C. H. Chao, S. H. Lin, W.-K. Liu, and P. Rentzepis, in TimeResolved Diffraction, edited by J. Helliwell and P. Rentzepis (Oxford University, New York, 1997), p. 260.

[7] A. Debnarova, S. Techert, and S. Schmatz, J. Chem. Phys. 125, 224101 (2006).

[8] S. L. Johnson, P. Beaud, E. Vorobeva, C. J. Milne, E. D. Murray, S. Fahy, and G. Ingold, Phys. Rev. Lett. 102, 175503 (2009).

[9] J. Cao and K. R. Wilson, J. Phys. Chem. A 102, 9523 (1998).

[10] N. E. Henriksen and K. B. Møller, J. Phys. Chem. B 112, 558 (2008).

[11] P. J. Ho and R. Santra, Phys. Rev. A 78, 053409 (2008).

[12] S. Tanaka, V. Chernyak, and S. Mukamel, Phys. Rev. A 63, 063405 (2001).

[13] S. Tanaka, S. Volkov, and S. Mukamel, J. Chem. Phys. 118, 3065 (2003).

[14] D. A. Kohl and E. J. Shipsey, Z. Phys. D 24, 39 (1992).

[15] I. Waller and D. R. Hartree, Proc. R. Soc. London A 124, 119 (1929).

[16] E. L. Saldin, E. A. Schneidmiller, and M. V. Yurkov, Opt. Commun. 148, 383 (1998).

[17] L. Van Hove, Phys. Rev. 95, 249 (1954).

[18] M. Altarelli et al., Tech. Rep., DESY, 2007 (unpublished), http://xfel.desy.de/tdr/tdr.
[19] T. Tschentscher, Chem. Phys. 299, 271 (2004).

[20] M. Born and K. Huang, Dynamical Theory of Crystal Lattices (Clarendon, Oxford, 1954).

[21] J. H. Hubbell, Wm. J. Veigele, E. A. Briggs, R. T. Brown, D. T. Cromer, and R. J. Howerton, J. Phys. Chem. Ref. Data 4, 471 (1975).

[22] M. Christensen, K. Haldrup, K. Bechgaard, R. Feidenhansl, Q. Kong, M. Cammarata, M. L. Russo, M. Wulff, N. Harrit, and M. M. Nielsen, J. Am. Chem. Soc. 131, 502 (2008).

[23] W.-K. Liu and S. H. Lin, Phys. Rev. A 55, 641 (1997).

[24] J. H. Shirley, Phys. Rev. 138, B979 (1965).

[25] H. Sambe, Phys. Rev. A 7, 2203 (1973).

[26] S. Guérin, F. Monti, J.-M. Dupont, and H. R. Jauslin, J. Phys. A 30, 7193 (1997).

[27] K. Drese and M. Holthaus, Eur. Phys. J. D 5, 119 (1999).

[28] B. Friedrich and D. Herschbach, Phys. Rev. Lett. 74, 4623 (1995).

[29] A. Messiah, Quantum Mechanics II (North-Holland, Amsterdam, 1962).

[30] T. Helgaker, P. Jørgensen, and J. Olsen, Molecular ElectronicStructure Theory (Wiley, New York, 2000).

[31] M. Takahashi, N. Watanabe, Y. Wada, S. Tsuchizawa, T. Hirosue, H. Hayashi, and Y. Udagawa, J. Electron Spectrosc. Relat. Phenom. 112, 107 (2000).

[32] J. F. Ying and K. T. Leung, J. Chem. Phys. 100, 7120 (1994).

[33] R. C. Ulsh, H. F. Wellenstein, and R. A. Bonham, J. Chem. Phys. 60, 103 (1974).

[34] R. S. Barbieri and R. A. Bonham, Phys. Rev. A 44, 7361 (1991).

[35] S. P. A. Sauer (private communication).

[36] M. Inokuti, Rev. Mod. Phys. 43, 297 (1971). 